\title{
Response of empty and fluid-filled, submerged spherical shells to plane and spherical, step-exponential acoustic waves
}

\author{
Michael A. Sprague* and Thomas L. Geers \\ Center for Acoustics, Mechanics and Materials, \\ Department of Mechanical Engineering, University of \\ Colorado, Boulder, C0 80309-0427, USA
}

Received 29 October 1998

Revised 26 April 1999

The title problem is solved through extension of a method previously formulated for plane step-wave excitation, which employs generalized Fourier series augmented by partial closure of those series at early time. The extension encompasses both plane and spherical incident waves with step-exponential pressure profiles. The effects of incident-wave curvature and profile decay rate on response behavior are examined. A method previously developed for assessing the discrepancy between calculated and measured response histories is employed to evaluate the convergence of the truncated series solutions. Also studied is the performance of doublyasymptotic approximations. Finally, the efficacy of modified Cesàro summation for improving the convergence of series solutions is examined. The documented computer program that produced the numerical results appearing in this paper, SPHSHK/MODSUM, may be down-loaded from the Web site http://saviac.xservices.com.

Keywords: Fluid-structure interaction, underwater shock, doubly-asymptotic approximations, benchmark solutions

\section{Introduction}

A series solution for the response of an empty submerged spherical shell excited by a plane stepexponential wave was first presented by Huang [11], and was extended by Huang et al. [12] to include the response due to a spherical step-exponential incident wave. Later, Zhang and Geers [16] employed convergence-enhancement techniques to obtain series

\footnotetext{
${ }^{*}$ Corresponding author.
}

solutions for the response of a fluid-filled or empty submerged spherical shell excited by a plane stepwave; these techniques are partial series closure at early time and modified Cesàro summation over all time [1]. Partial series closure consists of separating the early-time response into a closed-form portion and a complementary mode-sum portion. The closedform portion invokes the plane-wave approximation for the fluid-structure interaction and neglects stiffness effects in the shell. Cesàro summation is a modal filtering technique that substantially reduces Gibbs oscillations produced by a finite series representation of a discontinuous function. The FORTRAN program, SPHSHK/MODSUM, which was used to generate numerical results in Zhang and Geers [16], was made available for public use through the Shock and Vibration Information Analysis Center [7]. Most recently, Huang and Mair [13] extended Huang's 1969 formulation to include Cesàro summation of the first 70 terms of the series solution.

This paper extends the formulation of Zhang and Geers [16] to include excitation by plane step-exponential and spherical step-exponential incident waves. Standard Fourier summation with partial closure is used to generate results. Representative shell responses are shown for several incident-wave curvatures and decay profiles. Convergence of shell-response series solutions for these excitations is assessed with the aid of comprehensive error-factors [5,6]. In addition, the performance of the second-order doubly-asymptotic approximation $\left(\mathrm{DAA}_{2}\right)$ for treating the fluid-structure interaction (FSI) is examined [4]. Finally, problems associated with using modified Cesàro summation instead of standard Fourier summation are discussed.

\section{Problem description}

Figure 1 shows a schematic of a spherical shell about to be excited by an incident spherical wave. The shell is 


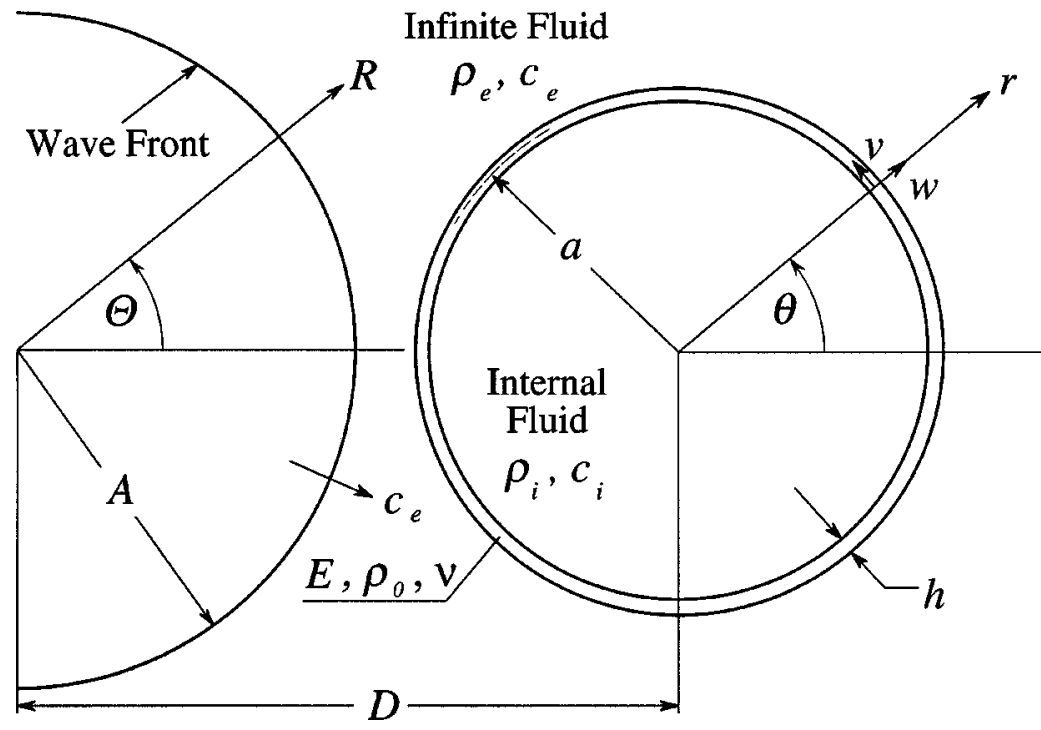

Fig. 1. Schematic of shell geometry and incident spherical wave with associated variables.

composed of an isotropic material with Young's modulus $E$, density $\rho_{0}$, and Poisson's ratio $\nu$. The shell thickness-to-radius ratio $h / a$ is assumed to be sufficiently small that thin-shell theory applies. Shell spherical coordinates and a nondimensional formulation are used throughout this paper. Length is normalized to the middle-surface shell radius $a$ and pressure is normalized to $\rho_{\mathrm{e}} c_{\mathrm{e}}^{2}$, where $\rho_{\mathrm{e}}$ and $c_{\mathrm{e}}$ are the mass density and speed of sound of the external fluid, respectively; the internal fluid is described by density $\rho_{\mathrm{i}}$ and soundspeed $c_{\mathrm{i}}$. Time is normalized to $a / c_{\mathrm{e}}$, which has the incident wave front moving over the shell during the nondimensional interval $0<t<2$. Radial and meridional displacements of the shell are given by $w(\theta, t)$ and $\nu(\theta, t)$, respectively.

The pressures for the internal wave, scattered external wave, and incident external wave are denoted by $p^{\mathrm{i}}(r, \theta, t), p^{\mathrm{s}}(r, \theta, t)$, and $p^{0}(r, \theta, t)$, respectively. The incident wave is described using an alternate spherical coordinate $(R, \Theta)$ system with the source located at the wave center, which is located a distance $D$ from the shell center. The wave expands radially at the fluid sound speed and has an instantaneous radius $A(t)$. The plane wave may be taken as a specialization of the spherical wave as $A \rightarrow \infty$.

\section{Formulation}

We follow the approach of Zhang and Geers [16]. For $0<t<2$, the shell velocities and surface pres- sures are expressed as

$$
\begin{aligned}
& \dot{\nu}(\theta, t)=-\sum_{n=1}^{N} \dot{\nu}_{n}(t) \frac{\mathrm{d}}{\mathrm{d} \theta} P_{n}(\cos \theta), \\
& \dot{w}(\theta, t)=\dot{w}^{*}(\theta, t)+\sum_{n=0}^{N} \dot{w}_{n}^{+}(t) P_{n}(\cos \theta), \\
& \underline{p}^{\mathrm{e}}(\theta, t)=\underline{p}^{0}(\theta, t)+\underline{p}^{\mathrm{s}^{*}}(\theta, t)+\sum_{n=0}^{N} \underline{p}_{n}^{\mathrm{s}^{+}}(t) P_{n}(\cos \theta), \\
& \underline{p}^{\mathrm{i}}(\theta, t)=\underline{p}^{\mathrm{i}}{ }^{*}(\theta, t)+\sum_{n=0}^{N} \underline{p}_{n}^{\mathrm{i}^{+}}(t) P_{n}(\cos \theta),
\end{aligned}
$$

where $p^{\mathrm{e}}$ and $p^{\mathrm{i}}$ are the external and internal pressures, respectively, an underscore denotes evaluation at the shell surface, $p^{0}$ is the external pressure associated with the incident acoustic wave, $P_{n}()$ is the Legendre polynomial of degree $n$, an asterisk denotes an initialresponse quantity, a plus indicates a complementary response quantity, an overdot denotes a time derivative, and $N$ is the maximum mode index of summation. For $t \geqslant 2$, Fourier summation alone gives satisfactory convergence; the counterparts to (1) are then (1) with $w^{*}(\theta, t)=p^{*}(\theta, t)=0$ and with the ${ }^{+}$superscripts removed. The incident radial fluid velocity $\dot{u}^{0}$ is related to the pressure $p^{0}$ by

$$
\dot{u}^{0}(r, \theta, t)=-\frac{\partial}{\partial r} \int_{0}^{t} p^{0}(r, \theta, t) \mathrm{d} t .
$$




\subsection{Plane step-exponential wave}

For a plane step-exponential wave, the incident pressure is given by

$p^{0}(r, \theta, t)=P_{\mathrm{I}} \mathrm{e}^{-\beta(t-r \cos \theta-1)} H(t-r \cos \theta-1)$,

where $P_{\mathrm{I}}$ is the peak value, $\beta$ is the decay constant, and $H()$ is the Heaviside step function. From (2), the associated radial fluid velocity is

$$
\begin{aligned}
\underline{\dot{u}}^{0}(r, \theta, t)= & P_{\mathrm{I}} \cos \theta \mathrm{e}^{-\beta(t-r \cos \theta-1)} \\
& \times H(t-r \cos \theta-1) .
\end{aligned}
$$

Evaluating (3) and (4) at the shell surface, we obtain

$$
\begin{aligned}
\underline{p}^{0}(\theta, t)= & P_{\mathrm{I}} \mathrm{e}^{-\beta(t-\cos \theta-1)} H(t-\cos \theta-1), \\
\underline{\dot{u}}^{0}(\theta, t)= & P_{\mathrm{I}} \cos \theta \mathrm{e}^{-\beta(t-\cos \theta-1)} \\
& \times H(t-\cos \theta-1) .
\end{aligned}
$$

The ordinary differential equations (ODEs) for the closed-form initial solution are given in Zhang and Geers [16] as

$$
\begin{aligned}
& \ddot{w}^{*}+v \dot{w}^{*}=-\mu\left(\underline{p}^{0}-\underline{\dot{u}}^{0}\right), \\
& \underline{p}^{\mathrm{s}^{*}}=\dot{w}^{*}-\underline{\dot{u}}^{0}, \\
& \underline{p}^{\mathrm{i}^{*}}=-\left(c_{\mathrm{i}} / c_{\mathrm{e}}\right) \dot{w}^{*},
\end{aligned}
$$

where $\mu=\left(\rho_{\mathrm{e}} / \rho_{0}\right)(a / h)$ and $v=\mu\left(1+\rho_{\mathrm{i}} c_{\mathrm{i}} / \rho_{\mathrm{e}} c_{\mathrm{e}}\right)$. The closed-form initial solution for radial shell velocity is found by solving the first of (6) for an incident wave defined by (5) with quiescent initial conditions. This solution is

$$
\begin{gathered}
\dot{w}^{*}(\theta, t)=\mu P_{\mathrm{I}}(1-\cos \theta)\left(\frac{\mathrm{e}^{-\beta(t-\cos \theta-1)}}{\beta-v}\right. \\
\left.-\frac{\mathrm{e}^{-v(t-\cos \theta-1)}}{\beta-v}\right) H(t-\cos \theta-1) .
\end{gathered}
$$

The internal and scattered pressures can be found directly from the radial shell velocity using the last two of (6).

The closed-form initial responses $\dot{w}^{*}(\theta, t), p^{\mathrm{s}^{*}}(\theta, t)$, and $p^{\mathrm{i}^{*}}(\theta, t)$ may be separated into modal components by numerical integration of the modal-decomposition relation

$$
q_{n}(t)=\left(n+\frac{1}{2}\right) \int_{0}^{\pi} q(\theta, t) P_{n}(\cos \theta) \sin \theta \mathrm{d} \theta
$$

where $q(\theta, t)$ is an axisymmetric function and $q_{n}(t)$ is its $n$-th modal component. These are entered as forcing functions in the complementary-response equations given by Eq. (34) in Zhang and Geers [16], which were formulated by subtracting the modal components of (6) from their modal equations for complete response. The resulting system of ODEs is integrated numerically to yield the complementary responses denoted by a superscript ${ }^{+}$in (1).

After the wave front has moved past the shell, modal summation alone can be used to calculate shell response. This requires only that the appropriate values for the free-field modal pressure $\underline{p}_{n}^{0}(t)$ and modal radial fluid velocity $\underline{\dot{u}}_{n}^{0}(t)$ be provided. These are determined from the modal-decomposition relation (8) with $q(\theta, t)$ being $p^{0}(\theta, t)$ and then $\underline{u}^{0}(\theta, t)$ as given by $(5)$. For $t \geqslant 2, \overline{(8)}$ may be simplified by separating out the temporal component of the integral and using the change of variable $x=\cos \theta$, which yields

$$
\begin{aligned}
& \underline{p}_{n}^{0}(t)=P_{\mathrm{I}}\left(n+\frac{1}{2}\right) \mathrm{e}^{-\beta(t-1)} \int_{-1}^{1} \mathrm{e}^{\beta x} P_{n}(x) \mathrm{d} x, \\
& \underline{\dot{u}}_{n}^{0}(t)=P_{\mathrm{I}}\left(n+\frac{1}{2}\right) \mathrm{e}^{-\beta(t-1)} \int_{-1}^{1} \mathrm{e}^{\beta x} x P_{n}(x) \mathrm{d} x,
\end{aligned}
$$

for which analytical solutions are readily found.

\subsection{Spherical step-exponential wave}

We now apply the above procedure to a spherical incident wave. The free-field pressure for this wave is given in the source coordinate system by (Fig. 1)

$p^{0}(R, \Theta, t)=P_{\mathrm{I}} \frac{A_{\mathrm{s}}}{R} \mathrm{e}^{-\beta\left(t-R+A_{\mathrm{s}}\right)} H\left(t-R+A_{\mathrm{s}}\right)$,

where $A_{\mathrm{S}}$ is the source standoff (radius of the wave front when it first reaches the shell). We note that the pressure at the wave front is calibrated such that $p^{0}=P_{\mathrm{I}}$, when $R=A_{\mathrm{s}}$. Equation (10) may be transformed into the shell coordinate system using the law of cosines and geometric relations, which yield the following:

$$
\begin{aligned}
& R=\sqrt{r^{2}+D^{2}+2 D r \cos \theta}, \\
& A_{\mathrm{s}}=D-1 .
\end{aligned}
$$


The free-field pressure in the shell coordinate system at any point in the fluid is therefore

$$
\begin{aligned}
& p^{0}(r, \theta, t)=\frac{P_{\mathrm{I}}(D-1)}{\sqrt{r^{2}+D^{2}+2 r D \cos \theta}} \\
& \quad \times \mathrm{e}^{-\beta\left(t-1+D-\sqrt{r^{2}+D^{2}+2 r D \cos \theta}\right)} \\
& \quad \times H\left(t-1+D-\sqrt{r^{2}+D^{2}+2 r D \cos \theta}\right) .
\end{aligned}
$$

Using (2), one can show that the radial fluid velocity in the shell coordinate system is given by

$$
\begin{aligned}
& \dot{u}^{0}(r, \theta, t)=P_{\mathrm{I}}(D-1)(r+D \cos \theta) \\
& \quad \times\left(\frac{\mathrm{e}^{-\beta\left(t-1+D-\sqrt{r^{2}+D^{2}+2 r D \cos \theta}\right.}}{r^{2}+D^{2}+2 r D \cos \theta}\right. \\
& \left.\quad-\frac{\mathrm{e}^{-\beta\left(t-1+D-\sqrt{r^{2}+D^{2}+2 r D \cos \theta}\right.}-1}{\beta\left(r^{2}+D^{2}+2 r D \cos \theta\right)^{3 / 2}}\right) \\
& \quad \times H\left(t-1+D-\sqrt{r^{2}+D^{2}+2 r D \cos \theta}\right) .
\end{aligned}
$$

Evaluating (12) and (13) at the shell surface, we obtain

$$
\begin{aligned}
\underline{p}^{0}(\theta, t)= & \frac{P_{\mathrm{I}}(D-1)}{Z} \mathrm{e}^{-\beta(t-1+D-Z)} \\
& \times H(t-1+D-Z), \\
\underline{\dot{u}}^{0}(\theta, t)= & P_{\mathrm{I}}(D-1)(1+D \cos \theta) \\
& \times\left(\frac{\mathrm{e}^{-\beta(t-1+D-Z)}}{Z^{2}}-\frac{\mathrm{e}^{-\beta(t-1+D-Z)}}{\beta Z^{3}}\right) \\
& \times H(t-1+D-Z),
\end{aligned}
$$

where $Z=\left(1+D^{2}+2 D \cos \theta\right)^{1 / 2}$.

These equations for the pressure and radial fluid velocity may now be entered into the first of (6) to find the closed-form initial solutions for $0<t<2$. Solving for the radial shell velocity, we obtain

$$
\begin{aligned}
\dot{w}^{*}(\theta, t)= & \left\{\frac{\left(1-\mathrm{e}^{-v(t-1+D-Z)}\right)(1+D \cos \theta)}{v \beta Z^{3}}\right. \\
& +\frac{\mathrm{e}^{-v(t-1+D-Z)}-\mathrm{e}^{-\beta(t-1+D-Z)}}{\beta-v} \\
& \left.\times\left[\frac{1+D \cos \theta}{Z^{2}}-\frac{1}{Z}-\frac{1+D \cos \theta}{\beta Z^{3}}\right]\right\} \\
& \times \mu P_{\mathrm{I}}(D-1) H(t-1+D-Z) .
\end{aligned}
$$

We can then determine the initial solutions for internal and scattered pressure directly from (15) using the last two of (6). These responses are then separated into modal components by numerically performing the integration in (8).

The equations for incident modal pressure and modal radial velocity are found by numerically performing the integration in (8) with $q(\theta, t)$ being $\underline{p}^{0}(\theta, t)$ and then $\underline{\dot{u}}^{0}(\theta, t)$ as defined in (14). As with the plane step-exponential wave, this process may be simplified for $t \geqslant 2$ by separating out the temporal portion from the integral and using the change of variable $x=\cos \theta$. Thus, the incident modal pressure and modal radial velocity for $t \geqslant 2$ are given by

$$
\begin{aligned}
\underline{p}_{n}^{0}(t)= & \left(n+\frac{1}{2}\right) P_{\mathrm{I}}(D-1) \mathrm{e}^{-\beta(t-1+D)} \\
& \times \int_{-1}^{1} \mathrm{e}^{\beta Z} Z^{-1} P_{n}(x) \mathrm{d} x \\
\underline{\dot{u}}_{n}^{0}(t)= & P_{\mathrm{I}}\left(n+\frac{1}{2}\right)(D-1)\left[\mathrm{e}^{-\beta(t-1+D)} \int_{-1}^{1} \mathrm{e}^{\beta Z}\right. \\
& \times(1+D x)\left(\frac{1}{Z^{2}}-\frac{1}{\beta Z^{3}}\right) P_{n}(x) \mathrm{d} x \\
& \left.+\int_{-1}^{1} \frac{(1+D x) P_{n}(x)}{\beta Z^{3}} \mathrm{~d} x\right],
\end{aligned}
$$

which are evaluated numerically.

\section{Convergence analysis}

This section describes how a method formulated by Geers [5,6] may be used to evaluate quantitatively the level of convergence of series solutions. The method was originally developed for evaluating the accuracy of a calculated response relative to a valid measured response. In this study, the response obtained by summing the first eight modes $(N=7)$ is taken as the measured response, and the response obtained by summing the first five modes $(N=4)$ is taken as the calculated response. Inherent in this approach is the assumption that higher and higher modes contribute less and less to the response.

The magnitude, phase, and comprehensive error factors are defined in Geers [6] as 


$$
\begin{aligned}
M & =\sqrt{\psi_{c c} / \psi_{m m}}-1, \\
P & =1-\psi_{c m} / \sqrt{\psi_{c c} \psi_{m m}}, \\
C & =\sqrt{M^{2}+P^{2}}
\end{aligned}
$$

respectively, in which the correlation factors $\psi_{c c}, \psi_{m m}$ and $\psi_{c m}$ are given by

$$
\begin{gathered}
\psi_{c c}=T^{-1} \int_{0}^{T} c^{2}(t) \mathrm{d} t, \\
\psi_{m m}=T^{-1} \int_{0}^{T} m^{2}(t) \mathrm{d} t, \\
\psi_{c m}=T^{-1} \int_{0}^{T} m(t) c(t) \mathrm{d} t,
\end{gathered}
$$

where $T$ is the total time under consideration, $m(t)$ is the measured response, and $c(t)$ is the calculated response.

Figure 2 shows, for $T=10$, comprehensive error factors for calculated radial-velocity histories pertaining to an empty steel spherical shell excited by spherical step-exponential waves at several standoffs and for many decay constants. The thickness-to-radius ratio $h / a$ is 0.01 , the mass-density ratio $\rho_{0} / \rho_{\mathrm{e}}$ is 7.7 , and the sound-velocity ratio $c_{0} / c_{\mathrm{e}}$ is $(13.8)^{1 / 2}$, where the plate velocity $c_{0}$ is given by $c_{0}=\left[E / \rho_{0}\left(1-\nu^{2}\right)\right]^{1 / 2}$. As shown, all errors are below 0.045 , which indicates satisfactory modal convergence. Even so, the comprehensive error factor for $\beta=10, D / a=2$, is one hundred times that for $\beta=0, D / a=\infty$.

\section{Representative shell responses}

This section presents numerical results for steel spherical shells submerged in water that are either empty or filled with water. System parameters are the same as those used in the convergence analysis. Figure 3 shows pressure histories at $\theta=\pi$ on the shell's external surface (the point of first contact) for an empty spherical shell excited by plane stepexponential waves with various decay constants. Figures 4 and 5 show associated radial-velocity histories at $\theta=\pi$ and $\theta=0$, respectively. These figures demonstrate satisfactory convergence, and exhibit expected trends as $\beta \rightarrow 0$. Figure 6 shows radial-velocity histories for a filled shell excited by plane step-waves with various decay constants. These histories do not show the level of convergence exhibited by their counterparts for an empty shell during $0 \leqslant t<2$, but are well converged for $t \geqslant 2$.

Figure 7 shows external-pressure histories at $\theta=\pi$ for an empty shell excited by spherical step-exponential waves, all with $\beta=2$. This figure demonstrates adequate convergence for the three standoffs considered, and shows that standoff has a small effect on external pressure. Figures 8 and 9 show radial velocity histories for the empty shell at $\theta=\pi$ and $\theta=0$, respectively. Unlike the situation with pressure, variations in standoff cause large changes in radial-velocity response, most notably at $\theta=0$. The results demonstrate adequate convergence, but also show how modal convergence at $\theta=\pi$ suffers as standoff is decreased. As the source of an incident spherical wave approaches the shell, higher response modes become increasingly important and the effects of modal truncation are more pronounced. Figure 10 shows radial-velocity response of a filled shell. As with the plane-wave excitation, convergence of the filled-shell histories is not quite as satisfactory as that of the empty-shell histories.

\section{DAA calculations}

Many underwater-shock calculations for complex marine structures make use of a doubly asymptotic approximation to treat the fluid-structure interaction $[3,4$, 8-10,15]. First-order $\left(\mathrm{DAA}_{1}\right)$, second-order $\left(\mathrm{DAA}_{2}\right)$, and third-order $\left(\mathrm{DAA}_{3}\right)$ approximations have been formulated. As part of the present study, response histories for spherical incident waves based on the secondorder, operator-matched DAA $[4,9,10]$ were compared with their acoustically based counterparts. Representative comparisons are shown in Figs 11 and 12 at $\theta=\pi$ and $\theta=0$ respectively; two types of $\mathrm{DAA}_{2-}$ based radial-velocity histories are shown, one obtained by modal summation as discussed above and the other produced by the finite-element/boundary-element (FE/BE) software assembly USA-DYNA [2,14]. For the USA-DYNA calculations, a quarter-model of a spherical shell was constructed with 864 thick-shell finite elements with 8 corner nodes and the same number of wet-surface boundary elements.

We observe in Figs 11 and 12 that the acousticsbased and $\mathrm{DAA}_{2}$-based modal-summation histories are almost indistinguishable for both plane step-wave and spherical step-exponential-wave excitation at $\theta=\pi$ but show small late-time differences at $\theta=0$. There is excellent agreement at all times between the USA-DYNA calculations and the $\mathrm{DAA}_{2}$-based modal- 


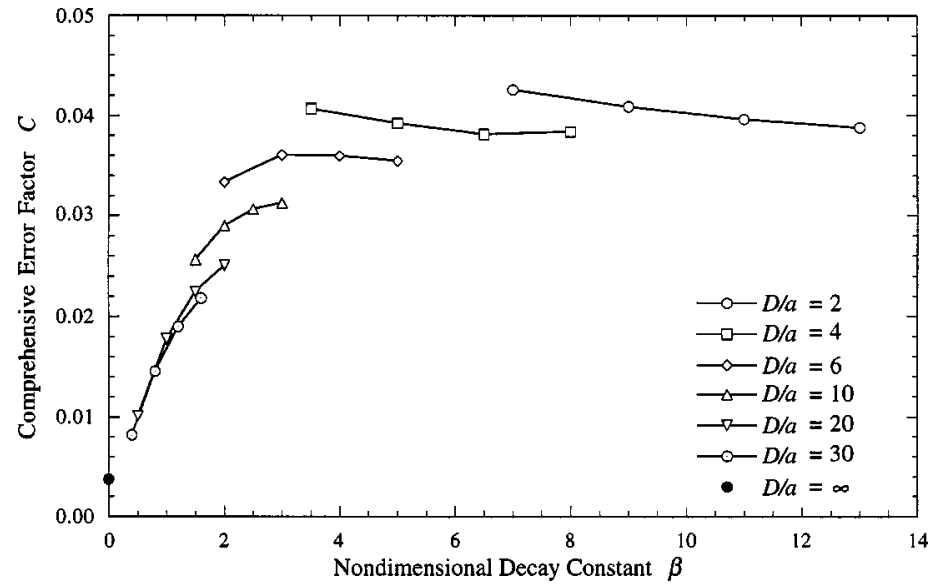

Fig. 2. Comprehensive error factors for radial-velocity histories at $\theta=\pi$ calculated by modal summation $(N=4$ vs $N=7)$; empty spherical shell excited by various spherical step-exponential waves.

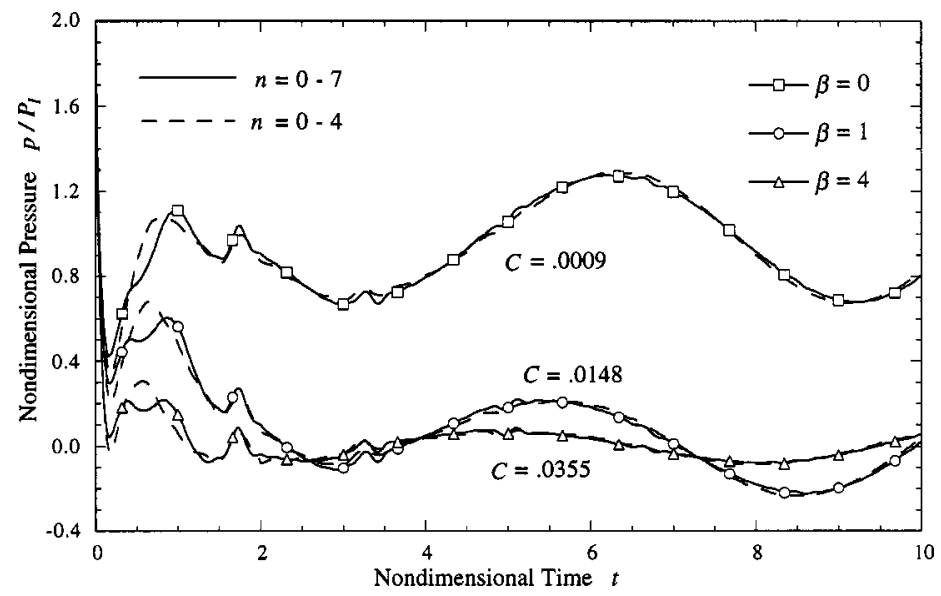

Fig. 3. External-surface pressure histories at $\theta=\pi$ for an empty spherical shell excited by plane step-exponential waves.

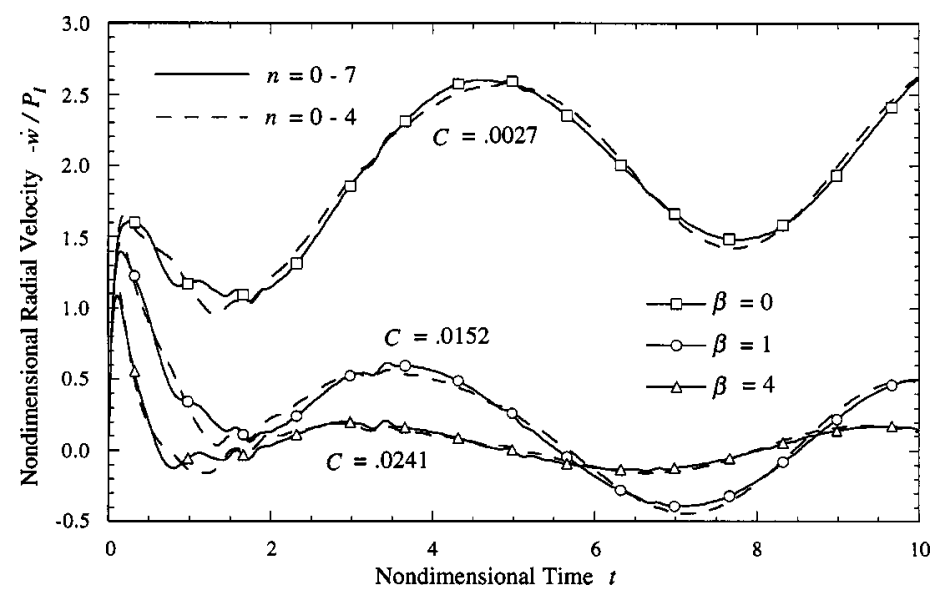

Fig. 4. Shell radial-velocity histories at $\theta=\pi$ for an empty spherical shell excited by plane step-exponential waves. 


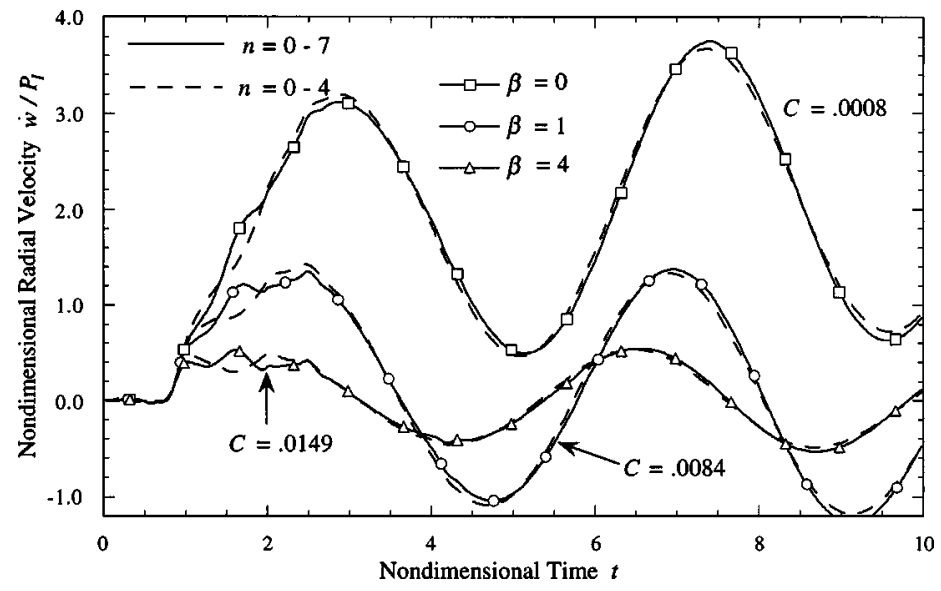

Fig. 5. Shell radial-velocity histories at $\theta=0$ for an empty spherical shell excited by plane step-exponential waves.

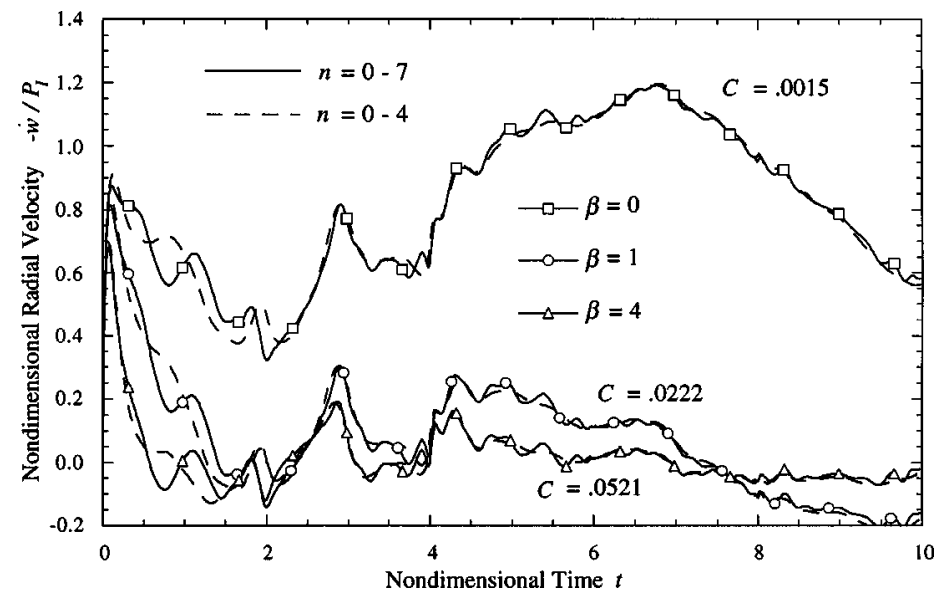

Fig. 6. Shell radial-velocity histories at $\theta=\pi$ for a filled spherical shell excited by plane step-exponential waves.

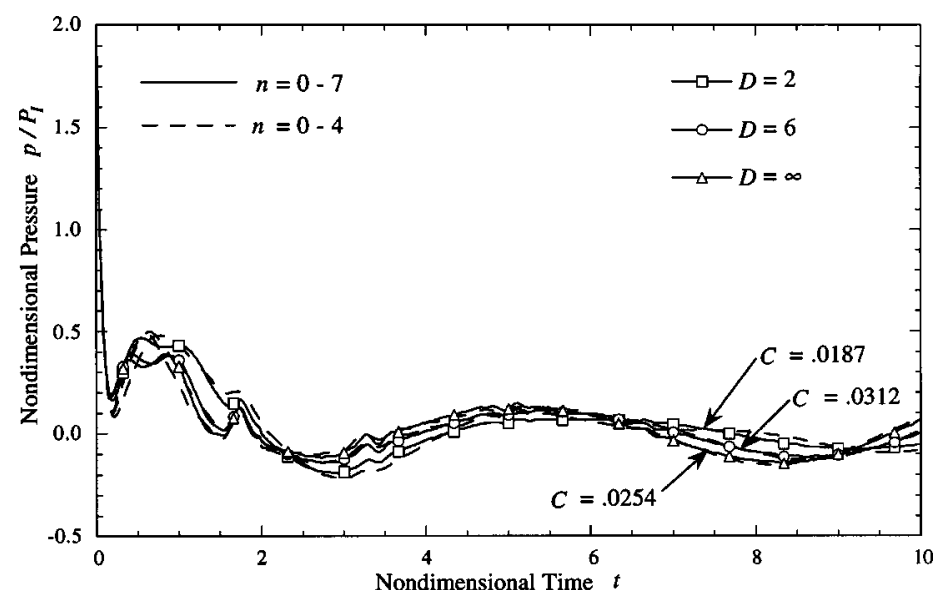

Fig. 7. External-surface pressure histories at $\theta=\pi$ for an empty spherical shell excited by spherical step-exponential waves $(\beta=2)$. 


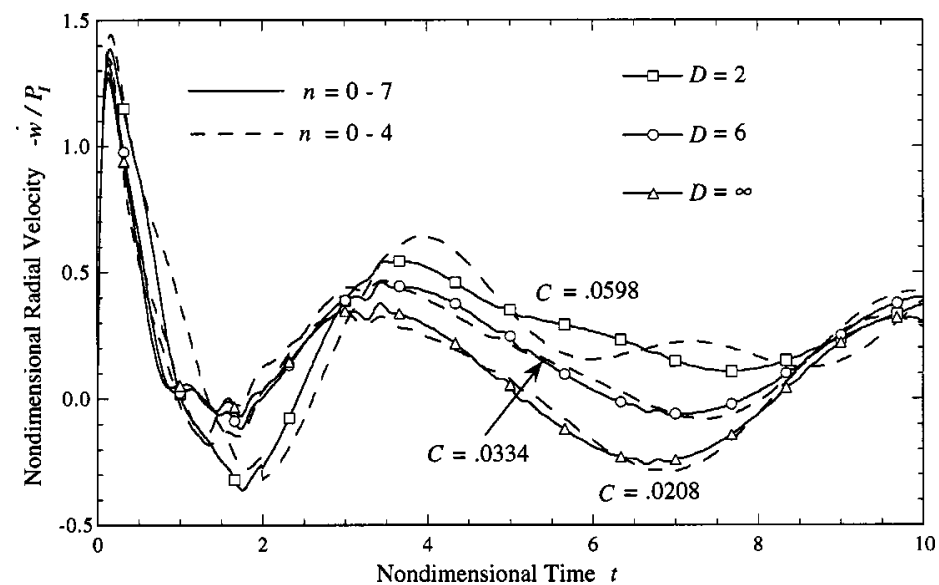

Fig. 8. Shell radial-velocity histories at $\theta=\pi$ for an empty spherical shell excited by spherical step-exponential waves $(\beta=2)$.

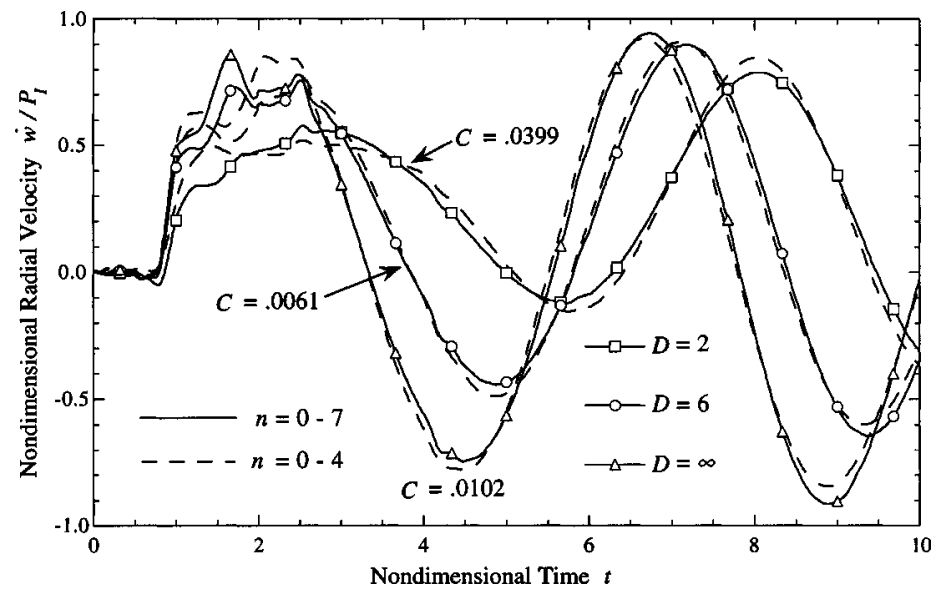

Fig. 9. Shell radial-velocity histories at $\theta=0$ for an empty spherical shell excited by spherical step-exponential waves $(\beta=2)$.

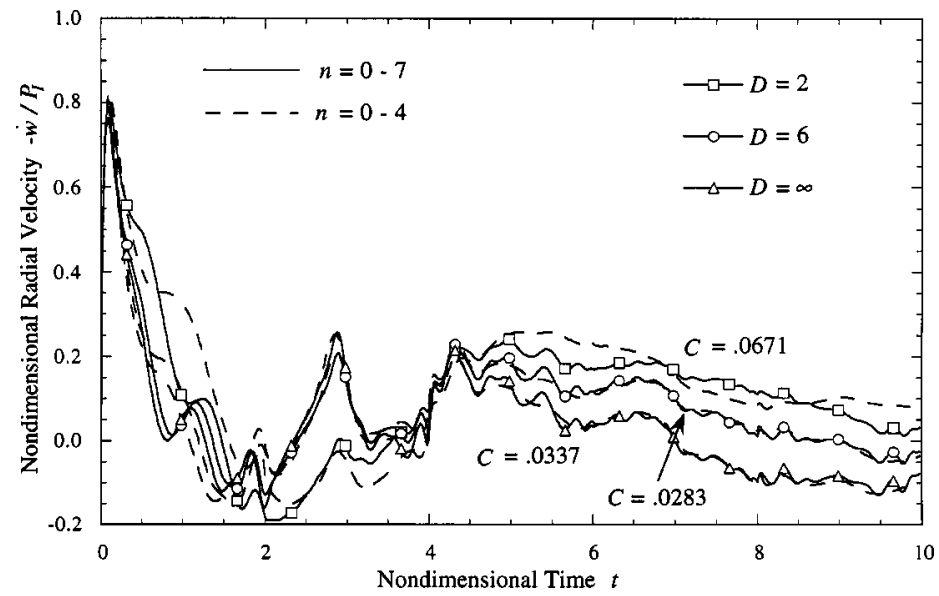

Fig. 10. Shell radial-velocity histories at $\theta=\pi$ for a filled spherical shell excited by spherical step-exponential waves $(\beta=2)$. 


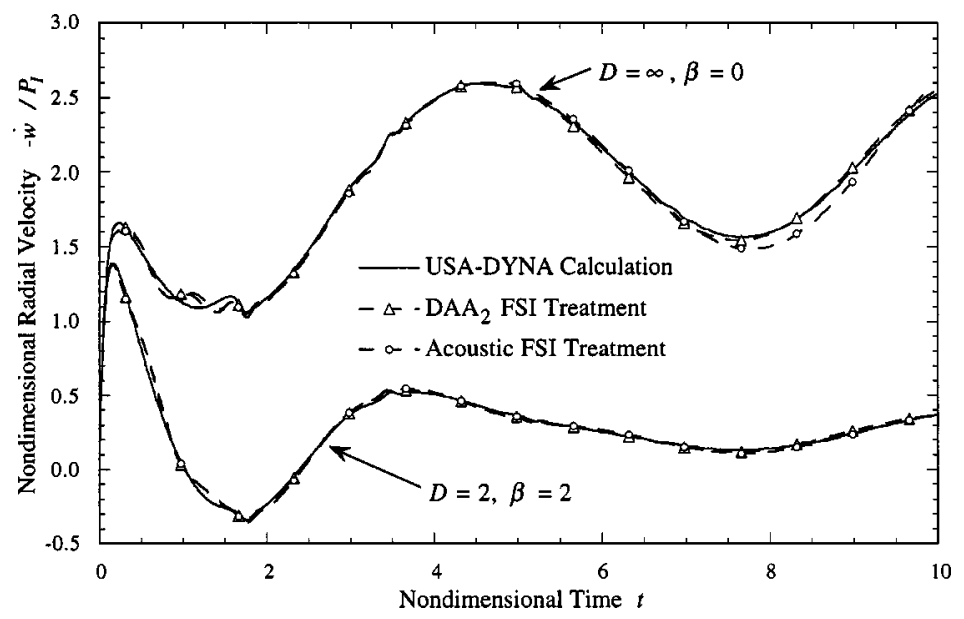

Fig. 11. Shell radial-velocity histories at $\theta=\pi$ for an empty spherical shell excited by a plane step wave $(D=\infty, \beta=0)$ and a spherical step-exponential wave $(D=2, \beta=2) ; N=7$.

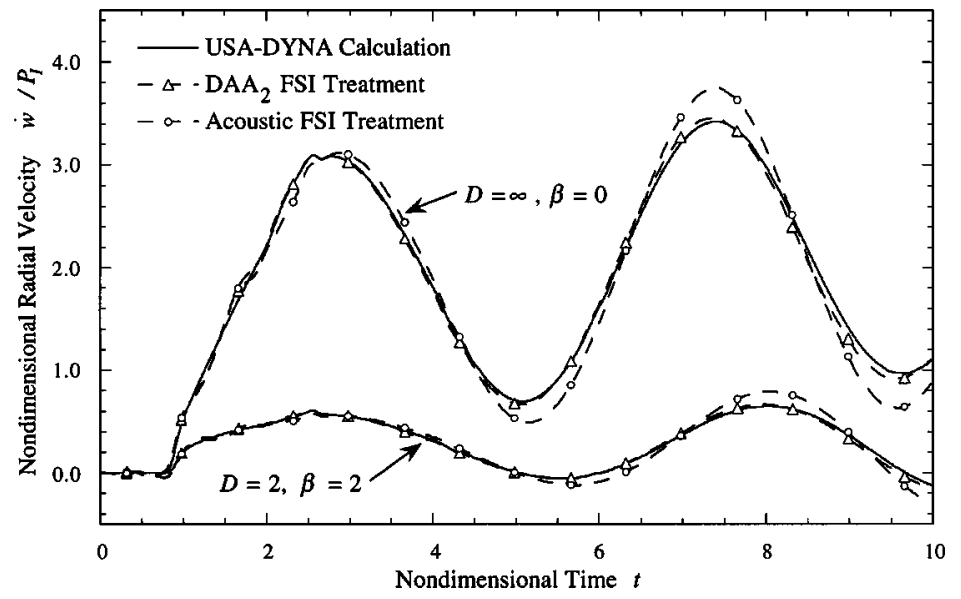

Fig. 12. Shell radial-velocity histories at $\theta=0$ for an empty spherical shell excited by a plane step wave $(D=\infty, \beta=0)$ and a spherical step-exponential wave $(D=2, \beta=2) ; N=7$.

summation histories at both points on the shell. This supports the conclusion that the series solutions for radial-velocity are adequately converged.

It should be noted that USA-DYNA calculations with 4-node shell elements produce unsatisfactory early-time radial-velocity histories at $\theta=\pi$. Figure 13 shows radial-velocity histories at $\theta=\pi$ due to excitation by a plane step-wave calculated with FE models consisting of 864 thick-shell elements ( 8 corner nodes), 864 shell elements (4 corner nodes), and 486 shell elements (again 4 corner nodes). The oscillatory 4-node-element histories differ significantly from the 8-node-element history during $0 \lesssim t \lesssim 0.6$. Such oscillations do not appear on the back of the shell, however. We see in figure, that refining the 4-node- elment mesh produces higher-frequency oscillations with slightly lower magnitude.

\section{Cesàro summation}

In order to improve convergence of their modalseries solutions, Zhang and Geers [16] and subsequently Huang and Mair [13] replaced the usual Fourier summation with Cesàro summation [1]. Although such replacement reduces Gibbs oscillations in a series representation of a discontinuous function, it can also slow the convergence rate when there are no discontinuities. This is demonstrated in Fig. 14, which shows incident-wave radial fluid-velocity histories at 


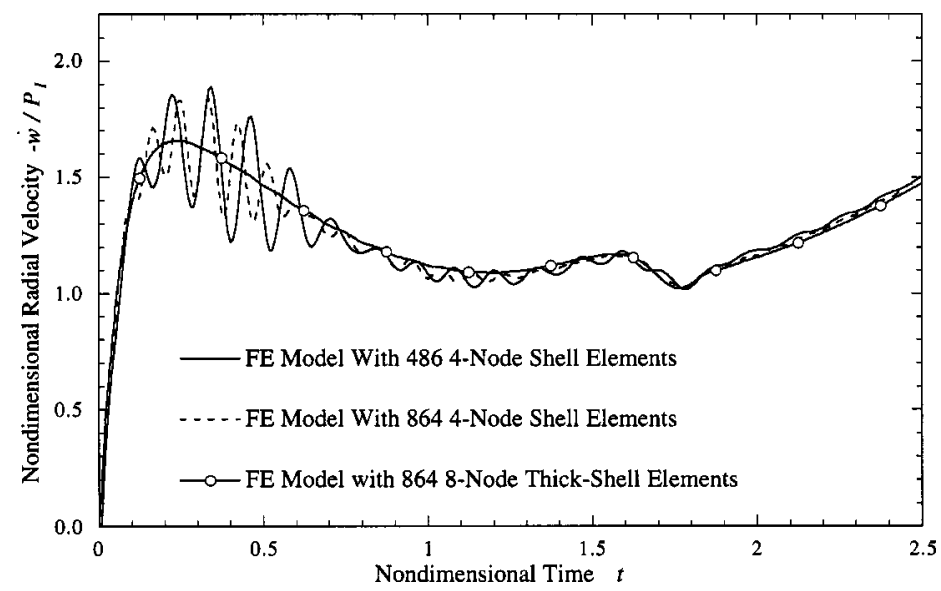

Fig. 13. Shell radial-velocity histories at $\theta=\pi$ for an empty spherical shell excited by a plane step wave $(D=\infty, \beta=0)$.

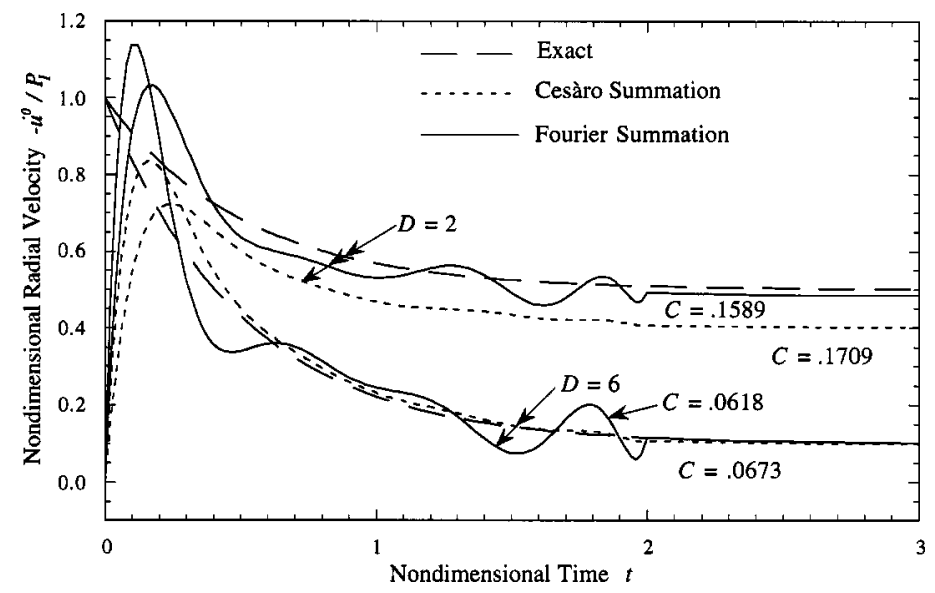

Fig. 14. Incident-wave radial-velocity histories at $r=1, \theta=\pi$ for several incident spherical waves with $\beta=2 ; N=7$.

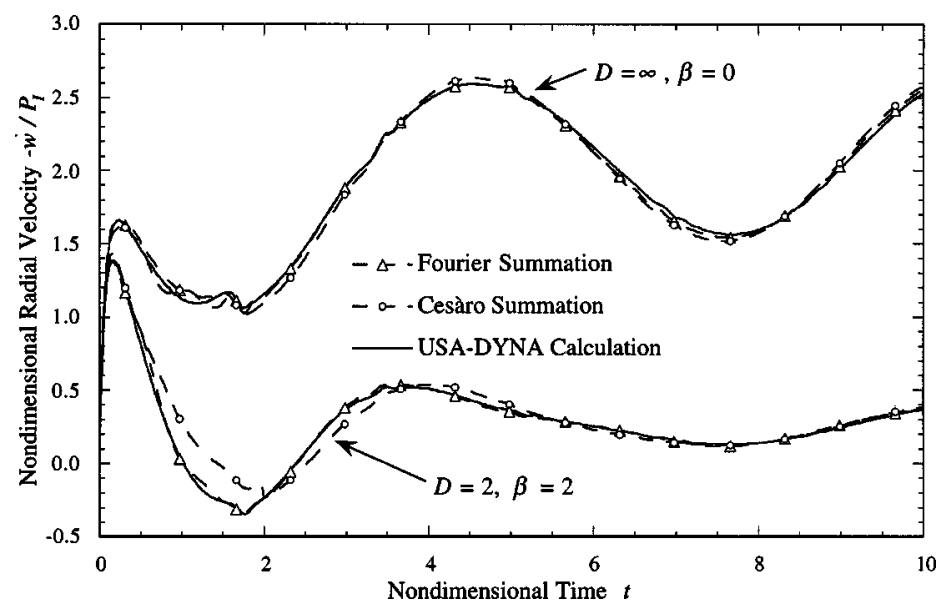

Fig. 15. DAA 2 -based shell radial-velocity histories at $\theta=\pi$ for an empty spherical shell excited by a plane step wave $(D=\infty, \beta=0)$ and a spherical step-exponential wave $(D=2, \beta=2) ; N=7$. 
$r=1, \theta=\pi$ produced by Fourier summation and by Cesàro summation. For large standoff $(D=6)$, Cesàro summation is clearly superior, in that the Gibbs oscillations at early time are subdued and convergence at late time is excellent. However, for small standoff $(D=2)$, Cesàro summation excessively attenuates early-time response and fails to converge satisfactorily at late time for the eight modes used; Fourier summation is clearly superior in this case.

As we have seen, partial closure of a Fourier series for shell response can greatly enhance modal convergence; hence, Cesàro summation is rarely needed and may be detrimental. This is illustrated in Fig. 15, which shows six $\mathrm{DAA}_{2}$-based velocity histories, two Cesàrosummed, two Fourier-summed, and two USA-DYNAcalculated. For the plane step-wave excitation, both the Fourier-summed and Cesàro-summed histories lie very close to the USA-DYNA-calculated history, but for excitation by the spherical step-exponential wave, the Cesàro-summed history departs somewhat from the other two.

\section{Conclusion}

We have extended the work of Zhang and Geers [16] to include spherical, step-exponential incident waves and have incorporated the extension in the program SPHSHK/MODSUM [7]. In doing so, we found satisfactory convergence of the series solutions by the usual Fourier summation, as long as partial series closure is employed. Such closure, however, is not adequate to treat discontinuous wet-surface and shell fields in the region $90^{\circ} \lesssim \theta \lesssim 155^{\circ}$ for standoffs substantially greater than the shell radius and in the region $120^{\circ} \lesssim \theta \lesssim 165^{\circ}$ for standoffs comparable to the shell radius [16]. With partial closure, Cesàro summation appears to be rarely, if ever, called for, and may impair series convergence.

\section{Acknowledgments}

The authors thank Mr. Thomas Littlewood for his help in the use of USA/LS-DYNA3D. This work was sponsored by the Defense Threat Reduction Agency under contract DNA-001-94-C-0004 with Mr. Michael Giltrud acting as technical monitor.

\section{References}

[1] T.M. Apostol, Mathematical Analysis: A Modern Approach to Advanced Calculus, Addison-Wesley, MA, 1957.

[2] J.A. DeRuntz, Jr. , The underwater shock analysis code and its applications, in: Proc. of the 60th Shock and Vibration Symposium, Virginia Beach, VA, 1989.

[3] T.L. Geers, Residual potential and approximate methods for three-dimensional fluid-structure interaction problems, J. of the Acoustical Society of America 49 (1971), 1505-1510.

[4] T.L. Geers, Doubly asymptotic approximation for transient motions of submerged structures, J. of the Acoustical Society of America 64 (1978), 1500-1508.

[5] T.L. Geers, An objective error measure for the comparison of calculated and measured transient response histories, The Shock and Vibration Bulletin 54 (1984), 99-107.

[6] T.L. Geers, Update of the comprehensive error factors, Shock and Vibration (1999), submitted.

[7] T.L. Geers and T. Ju, A computer program for a canonical problem in underwater shock, Shock and Vibration 1(4) (1994), 331-337.

[8] T.L. Geers and B.T. Toothaker, Evaluation of doubly asymptotic approximations for boundary element analysis in transient acoustics, in: Fundamental Solutions in Boundary Elements, Formulation and Integration, F.G. Benitez, ed., 1997, pp. 139156.

[9] T.L. Geers and P. Zhang, Doubly asymptotic approximations for submerged structures with internal fluid volumes: Formulation, J. of Applied Mechanics 61 (1994), 893-899.

[10] T.L. Geers and P. Zhang, Doubly asymptotic approximations for submerged structures with internal fluid volumes: Evaluation, J. of Applied Mechanics 61 (1994), 900-906.

[11] H. Huang, Transient interaction of plane acoustic waves with a spherical elastic shell, J. of the Acoustical Society of America 45(3) (1969), 661-670.

[12] H. Huang, Y.P. Lu and Y.F. Wang, Transient interaction of spherical acoustic waves and a spherical elastic shell, J. of Applied Mechanics 38 (1971), 71-74.

[13] H. Huang and H.U. Mair, Neoclassical solution of transient interaction of plane acoustic waves with a spherical elastic shell, Shock and Vibration 3(2) (1996), 85-98.

[14] LS-DYNA user's manual: Nonlinear dynamic analysis of structures in three dimensions, Version 940, Livermore Software Technology Corporation, Report No. 1082, 1997.

[15] B. Nicolas-Vullierme, A contribution to doubly asymptotic approximations: An operator top-down approach, in: Numerical Techniques in Acoustic Radiation, NCA-Vol. 6, ASME, New York, 1989, pp. 7-13; also, J. of Vibration and Acoustics 113 (1991), 409-415.

[16] P. Zhang and T.L. Geers, Excitation of a fluid-filled, submerged spherical shell by a transient acoustic wave, J. of the Acoustical Society of America 93(2) (1993), 696-705. 

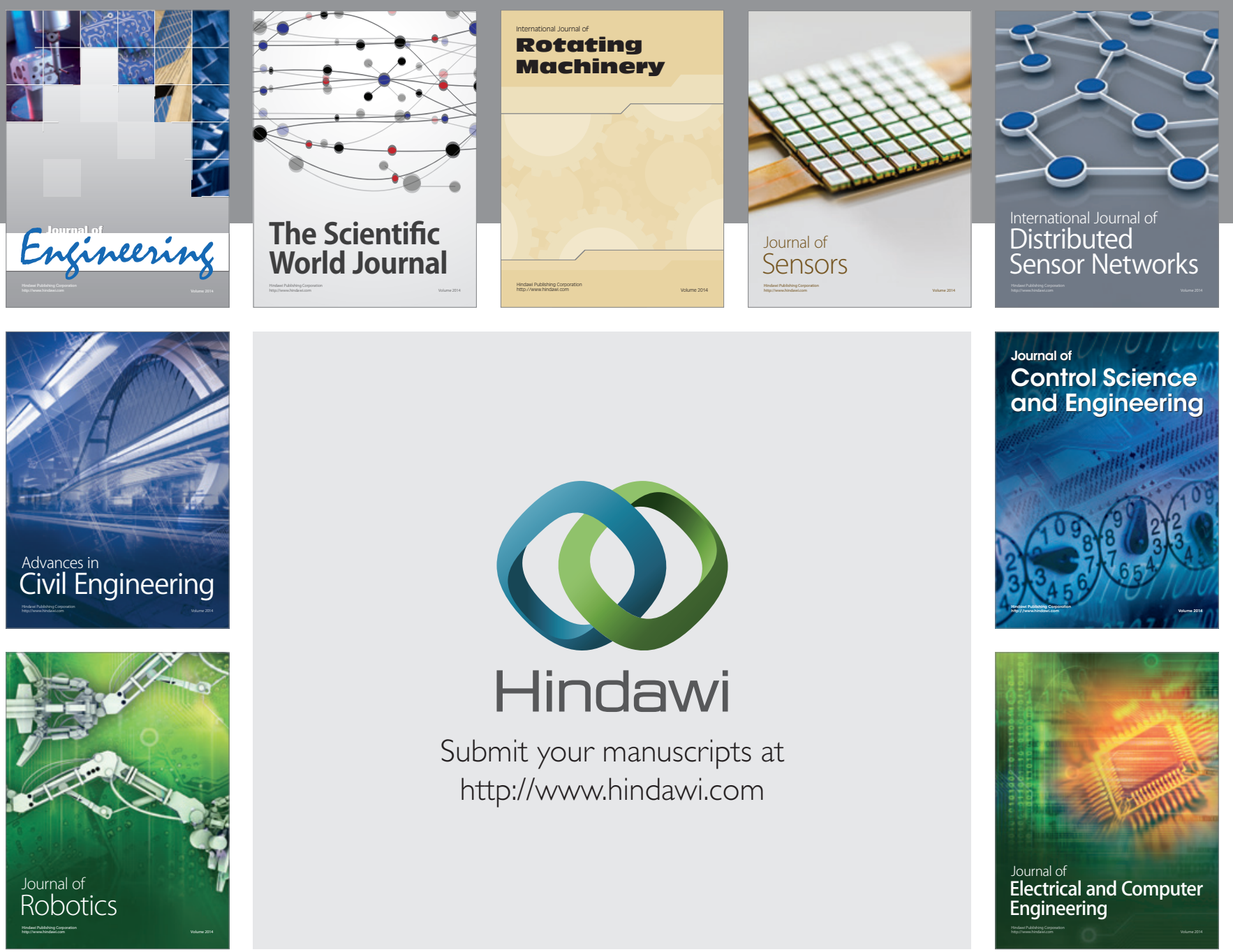

Submit your manuscripts at

http://www.hindawi.com
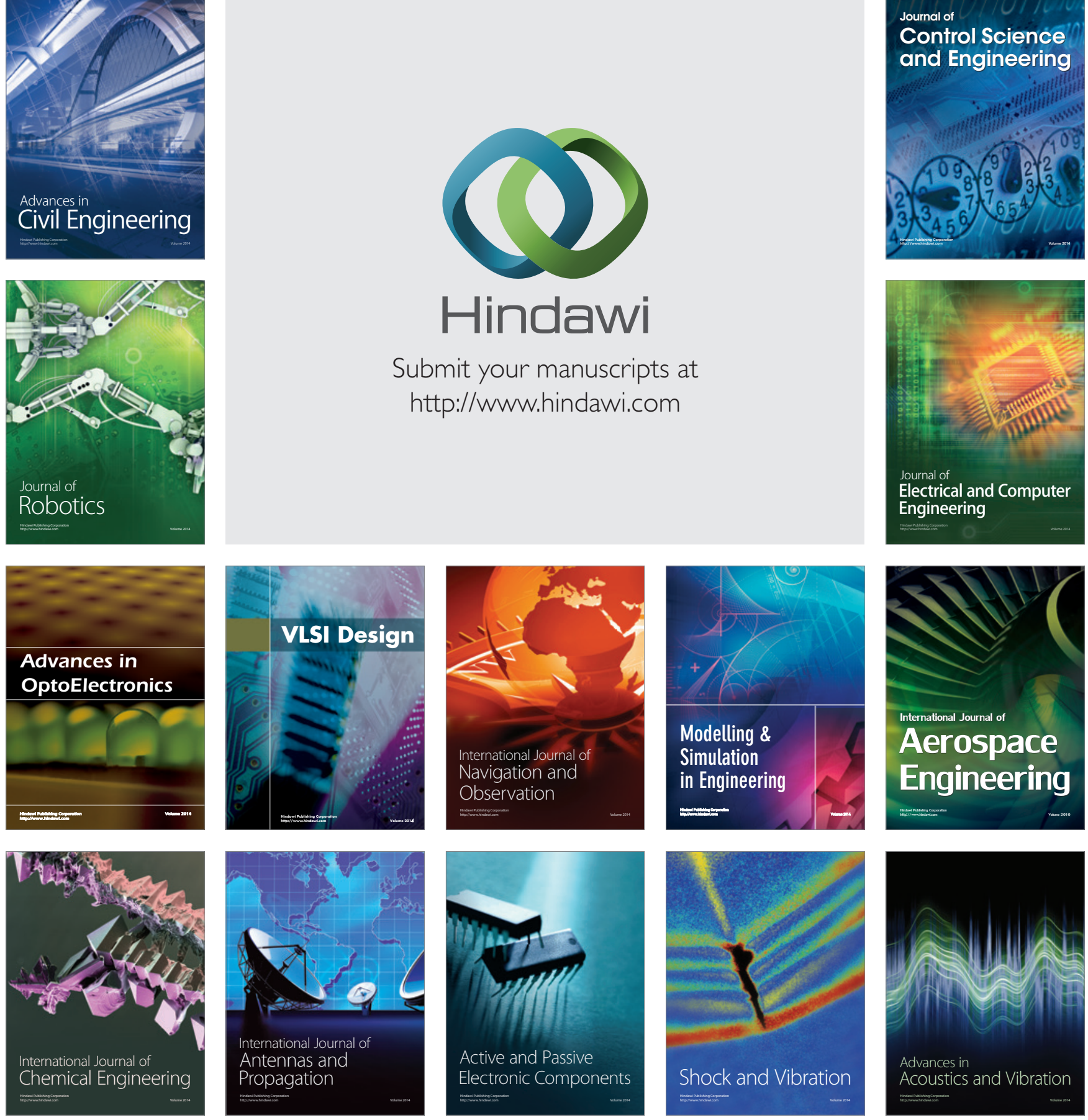